\title{
Homology Modelling of Human P-glycoprotein
}

Laura Domicevica and Philip C. Biggin*

Department of Biochemistry, University of Oxford, South Parks Road, Oxford, OX1 3QU, United Kingdom.

Author emails:

LD: laura.domicevica@bioch.ox.ac.uk

PCB: philip.biggin@bioch.ox.ac.uk

*To whom correspondence should be addressed.

Email: philip.biggin@bioch.ox.ac.uk

Tel. +441865613305

Fax. +441865 613238

Biochemical Society Transactions

Keywords: Molecular Dynamics, ABC Transporters, multidrug resistance, simulation, export pump, computational

Abbreviations: ATP, adenosine triphosphate; MDR1, multidrug resistance protein 1; NBD, nucleotide binding domain; P-gp, P-glycoprotein; PMF, potential of mean force; POPC, 1-palmitoyl-2-oleoyl-phosphatidyl-choline; $\mathrm{RMSD}$, root mean square deviation; TMD, transmembrane domain; $\mathrm{TMH}$, transmembrane helix. 


\begin{abstract}
P-glycoprotein is an ATP-binding cassette transporter that exports a huge range of compounds out of cells and is thus one of the key proteins in conferring multidrug resistance in cancer. Understanding how it achieves such a broad specificity and the series of conformational changes that allow export to occur form major, on-going, research objectives around the world. Much of our knowledge to date has been derived from mutagenesis and assay data. However, in recent years, there has also been great progress in structural biology and although the structure of human P-glycoprotein has not yet been solved, there are now a handful of related structures on which homology models can be built to aid in the interpretation of the vast amount of experimental data that currently exists. Many models for P-gp have been built with this aim, but the situation is complicated by the apparent flexibility of the system and by the fact that although many potential templates exist, there is large variation in the conformational state in which they have been crystallized. In this review, we summarize how this approach has been used in the past, how models are typically selected and finally illustrate how molecular dynamics simulations can be used as a means to give more confidence about models that have been generated via this approach.
\end{abstract}




\section{Introduction}

In recent years there has been substantial progress in using structural and computational methods to improve our understanding of multidrug efflux mechanisms [1] [2]. However, by far the most studied is P-glycoprotein. Pglycoprotein (P-gp), also known as multidrug resistance protein 1 (MDR1) or ATP-binding cassette sub-family B member 1 (ABCB1) is an ATP-dependent efflux pump with broad substrate specificity. It is thought to be one of the main reasons why tumor cells exhibit resistance to drugs and also plays a role in maintaining the blood brain barrier. It is able to export an extremely wide range of compounds [3] and that poses particular problems for the design of anti-cancer compounds, which may otherwise be extremely effective at their target. Current strategies to deal with this problem typically utilize knowledgebased methods on the back of structure-activity relationship data [3]. An understanding of the mechanism by which P-gp not only recognizes compounds, but also how it exports them should improve our prospects for designing compounds that are not susceptible to export by P-gp.

Although there is a vast amount of functional data, the lack of a crystal structure of human P-gp has hindered rational drug design strategies. However, in recent years, structures have started to appear for related proteins that can be used as templates for homology modelling to generate structure-based hypotheses, which in turn, can be tested experimentally.

There are currently a number crystal structures that can and have been used as templates for modelling human P-gp (Figure 1). One the first structures that was used as a template, was the Sav1866 transporter from $S$. aureus, which was crystallized in an outward-open state with both ADP [4] and nonhydrolysable ATP analogue bound [5]. Another outward-open structure that was solved subsequently was that of MsbA [6]. All the other structures solved so far are in some kind of inward-open state and come from a variety of different species (see Table 1 and Figure 1).

The publication of the C. elegans P-gp structure by Jin et al. [7] highlighted some differences with respect to the first mouse structures. The $C$. elegans structure was supported by arginine mutagenesis data and revealed that the helix register of transmembrane helix (TMH) 5 in the original mouse structure is likely not correct [8]. Several other crystal structures of mouse P-gp have been published since [9, 10], including a refined structure with additional experimental electron density maps that allowed the correct helical register to be modeled [11]. A human homology model from mouse crystal structure was used to dock several compounds in a flexible receptor showing different binding sites for large and small compounds [10,12].

Of course, as well as serving as templates for modelling human P-gp, these structures are of interest in their own right. The behaviour of mouse P-gp with substrate or inhibitor bound was investigated with molecular dynamics simulations showing that inhibitors keep nucleotide binding domains (NBD)s from approaching each other, while a bound substrate on the other hand would allow closure of the NBBs in a conformational more compatible with 
ATP hydrolysis [13]. O'Mara et al. assessed the impact of environment on protein stability [14], noticing that in a cholesterol-enriched 1-palmitoyl-2oleoyl-phosphatidyl-choline (POPC) bilayer, magnesium ions were required for a stable simulation of the mouse P-gp. However, this study was completed without the flexible linker present in the P-gp structure, a feature important in substrate specificity and drug transport $[15,16]$. In another molecular dynamics study with the same structure, the linker was built in to assess its importance in stabilization of the NBDs where it acts as a "damper" reducing the movements of the cytoplasmic regions of P-gp [17]. The transition pathway of MsbA was investigated with nonequilibrium-driven MD simulations revealing the highly cooperative nature of transmembrane domain (TMD) and NBD movement [18].

Presumably owing to it being one of the first structures solved and indeed is still considered an excellent template for the outward-facing state, the Sav1866 structure has been subjected to many MD studies [19-24]. Much can be gleaned from these studies in terms of general exporter function, but in this review, we focus specifically on models of P-gp and then illustrate how such models are typically made and evaluated.

\section{Modelling Studies}

One of the main goals or aims of homology modelling studies has been to build a model that can then be used to rationalize some existing structureactivity relationship (SAR) data [3, 25]. For example, dual inhibitors of P-gp and MRP1 were investigated via homology modelling of human P-gp based on the first mouse structure. Despite the sequence similarity between MRP1 and human P-gp it appears that there is enough sequence variation to give different geometric and physicochemical properties [26]. Palmeira et al. used homology models of P-gp in combination with docking to rationalize the P-gp sensitivity of aminated thioxanthone derivatives that have antitumor properties. They were able to show that derivatives could be designed that inhibited P-gp and also led to an improved in vitro efficacy in a sensitized drug-resistant (to doxorubicin) cell line [27]. Other studies have used docking to explore explored the binding of potential novel inhibitors such as 6(methylsulfinyl)hexyl isothiocyanate [28] and guanidine alkaloid [29]. They found in each case that these drugs bind in similar locations to known P-gp inhibitors such as QZ59-RRR [28] and verapamil [29].

Klepsch et al. explored the binding mode of propafenone derivatives in homology models derived from the Sav1866 and mouse crystal structures to capture the differences between the conformational states [30]. The binding modes were similar in both models - propafenone binding occurred on the $\mathrm{TMH}$ 5-8 interface overlapping part of the known cyclic inhibitor and verapamil-binding site. This work was followed by docking of benzopyrano[3,4-b][1,4]oxazines, which showed clustering of compounds close to TMHs 4,5 and 6 [31]. Interestingly, inhibitors whose structure-affinity pattern is affected by their ability to permeate the bilayer were distributed close to the entrance gate, while the other molecules could also be found 
deeper in the binding site. In a later study [32] they also found that benzophenones bind similarly to the previously investigated propafenone inhibitor.

A study investigating the structure-activity relationship of analogues of the third generation inhibitors, tariquidar and elacridar, in homology models based on mouse P-gp and Sav1866 crystal structures showed that their binding sites might overlap with the Hoechst 33342 and rhodamine binding sites and possibly interacts with both [33]. In another study involving tariquidar and propafenone derivatives, Jara et al. used molecular dynamics simulations and docking in a human homology model from the mouse crystal structure to estimate the binding energy of the modulators [34]. They concluded that hydrophobic interactions and molecular flexibility of the compounds are the main factors for inhibition. Although most studies have focused on the transmembrane cavity, other studies have examined the mode of action of compounds like piperine, which may act via the NBD [35].

\section{Studies that Incorporated Dynamics}

The interactions of $\mathrm{C}_{60}$ fullerene, a potential drug carrier, with $\mathrm{P}$-gp were investigated by a combination of in vitro methods and molecular dynamics simulations using a human homology model from the mouse structure inserted into a POPC bilayer [36]. The same model was used in a later study of paclitaxel and doxorubicin interactions with P-gp [37] where free diffusion of both drugs towards the binding site was observed in 100 ns of unbiased molecular dynamics simulations.

The crystal structure of $C$. elegans P-gp has been used to make homology models used in docking of anticancer compounds investigated by molecular dynamics simulations and free energy calculations [38]. One of the compounds, NSC745689, did not show any stable interactions with the binding site, suggesting that it is not a substrate of P-gp and this was confirmed with in vitro experiments.

A much more ambitious goal for P-gp modelling is to try to predict the transitions between conformational states. O'Mara and Tieleman [39] built a model of human P-gp based on the Sav1866 structure and, in combination with simulation data from MalK and BtuCD, proposed models of P-gp in the apo state. The transport mechanism of P-gp was also investigated using targeted MD on a homology model based on Sav1866 to obtain structures of intermediate states between the outward-open and inward-open conformations [40]. Docking of 21 substrates and inhibitors on 26 nonredundant transitional structures mostly agreed with previous biochemical evidence of the drug-binding site. Further work on these structures consisted of screening for selective compounds that would inhibit ATP hydrolysis by binding to NBDs and not by targeting the transmembrane binding-site [41]. 
The communication interface between the NBDs and the TMDs was investigated by homology modelling and molecular dynamics revealing hydrogen bond networks of conserved motifs in the NBDs and intracellular coupling helices [42]. Zeino et al. showed that even though it might be possible to discriminate between substrates, modulators and inhibitors of P-gp by molecular docking alone, care must be taken when interpreting the possible binding site position of a drug [43]. Pan and Aller [44] compared inward and outward models of P-gp with particular emphasis on the allosteric effect of ATP on the conformational dynamics. They also concluded that mouse P-gp and Sav1866 might employ slightly different transport mechanisms, especially with respect to the role of water. More recently, O'Mara and colleagues investigated potential of mean force (PMF) profiles for morphine and nicardipine and showed that they bind at different but overlapping sites within central transmembrane cavity [45].

\section{Using Modelling and Molecular Dynamics}

Though more X-ray data is now starting to appear, it is unlikely to provide a complete picture of the mechanism of export for P-gp, even if structures were solved in multiple states. To that end, it is clear that modelling can provide a useful way of generating new hypotheses that can be tested by other experimental methods. As currently, there is no structure for human P-gp in any state, one has to use homology modelling as the starting point. However, the situation is complicated now by the appearance of crystal structures from several different species in similar but not identical states. A key question is how we establish the quality of a model. In this part of the review we demonstrate a typical approach that can be used.

The resolution and sequence identity to the template crystal structure affect the resulting homology model. The crystal structures of membrane proteins are often obtained in the presence of detergents or micelles. This could be a particular issue for P-gp, due to its functional dependence on lipid species and localization [46]. Homology models inherit these flaws and must be further refined, for example, by molecular dynamics to ensure their stability in a membrane bound environment (see for example the work of O'Mara [14]). Although MD has been used in this way, one must remember that a complete sampling of all the conformational states is unlikely (typical current simulation times of are of the order of hundreds of nanoseconds) and even with extensive sampling, one still needs an appropriate method to define the quality of the model. Typically, the DOPE (Discrete Optimized Protein Energy) score is evaluated which relies on a statistical potential to evaluate model quality [47]. More simply, the model may be evaluated in terms of the number of residues that sit within the allowed regions of the Ramachandran plot and whilst this provides a good assessment of stereochemical quality, it does not provide any assessment of the likelihood that the structure resembles a native structure. For that, the QMEAN Z-score can be used [48]. When building models that could be derived from several different X-ray templates as is the case here, the Ramanchandran plot is often not particularly useful, as the resulting models all tend to have very similar scores. 


\section{P-gp Modeling}

In the case of P-gp, a compromise between sequence identity and resolution of the structure often has to be accepted, as the eukaryotic structures generally (with the exception of $A B C B 10$ ) have lower resolution than those of the bacterial homologues (Table 1). Models of human P-gp can be built on the available crystal structures and assessed in terms of their QMEAN Zscore to gain an idea of the overall quality of the models (Table 2). Since the QMEAN Z-score uses solvent accessibility as one of the scoring components, membrane proteins tend to yield poorer scores, predicting lower quality models than they are in reality [48]. However, this is a known limitation and the scores can provide a useful method to rank and compare very different models such as those from P-gp homologues in various conformational states.

For example, several high quality human homology models based on refined mouse [11], C. elegans [7] and C. merolae [49] ABCB1 crystal structures can be generated (See Supplementary Information for details). The QMEAN Zscores of these models ranged from -1.86 (best) to -2.14 (see Table 2). This result is comparable to a medium quality model (mean Z-score -1.75) as previously suggested without taking into account the unfavourable solvent accessibility term [48]. Interestingly, eukaryotic structures of ABCB1 provided better homology models than the structures of bacterial homologues or human $A B C B 10$, suggesting that high sequence identity is preferable for high quality models. However, the cut-off is not exact, as the templates of the best homology models show sequence identity to human P-gp ranging from 39 to $87 \%$.

It should be remembered that the homology model (and indeed the template structure) are single snapshots. In reality the protein is a dynamic object with conformational variability. A way to compare that is to use molecular dynamics. For example, models from the refined mouse, $C$. elegans and $C$. merolae crystal structures can be inserted into lipid bilayers (Figure 2A) and their dynamics monitored (See SI for details). In such simulations the dynamics of P-gp reflect its functional motion. The overall root mean squared deviation (RMSD) of the $\mathrm{C} \alpha$ atoms is fairly large for a membrane protein (Figure 2B), but this simply reflects the "breathing motions" of the protein as has also been described by other researchers using MD alongside double electron-electron resonance spectroscopy [50]. The predominant motion is the opening and closing motion that can best be summarized by the distance between the NBD domains (Figure 2C). As can be seen the model based on the $C$. elegans structure exhibits the most dramatic movements over the course of $100 \mathrm{~ns}$, and the model based on the refined mouse crystal structure exhibits the least fluctuation in this regard.

One aspect that modelling and MD simulation in particular could begin to address in the future are the questions surrounding drug access to the central binding cavity. There are two main routes that have been proposed; access 
directly from the cytoplasm or the more commonly accepted "hydrophobic vacuum cleaner model" where drugs enter from laterally from within the membrane (Figure 3). The latter route is generally accepted as being highly likely especially for non-polar compounds. In crystal structures and P-gp models, one can visualize distinct entrance portals into the central cavity (Figure 3B); one at the level of the membrane and one situated more towards the cytoplasm. However, during the course of MD simulations, the upper, membrane portals often appear to become occluded (Figure 3B). Thus, how compounds access the central drug-binding cavity remains unclear at this time and thus will require much more detailed investigations.

\section{Conclusions}

Consideration of P-gp is essential for many aspects of drug development. Despite excellent progress in recent years particularly in terms of structural biology, there are many key questions that are still open, and are likely to remain open even if we are fortunate enough to obtain a high-resolution structure of human P-gp itself. How is P-gp able to recognize such a diverse range of substrates and what makes a compound a likely substrate? What is the nature of the conformational cycle that pumps compounds out of the cell into the blood stream? How is that coupled to ATP hydrolysis? It is likely that a multidisciplinary approach will provide the best chance of answering these and related questions. There is no doubt that modelling and molecular dynamics simulations can, and will, play a huge role in helping to refine our understanding of this important protein.

\section{Acknowledgements}

LD thanks the NC3Rs for studentship support. 


\section{References}

1 Wong, K., Ma, J., Rothnie, A., Biggin, P. C. and Kerr, I. D. (2014) Towards understanding promiscuity in multidrug efflux pumps. Trends Biochem. Sci. 39, 8-16

2 Ferreira, R. J., Ferreira, M.-J. U. and dos Santos, D. J. V. A. (2015) Reversing cancer multidrug resistance: insights into the efflux by $A B C$ transports from in silico studies. Wiley Interdiscip. Rev. Comp. Mol. Sci. 5, 27-55

3 Chen, L., Li, Y., Yu, H., Zhang, L. and Hou, T. (2012) Computational models for predicting substrates or inhibitors of P-glycoprotein. Drug Discov. Today. 17, 343-351

4 Dawson, R. J. P. and Locher, K. P. (2006) Structure of a bacterial multidrug ABC transporter. Nature. 443, 180-185

5 Dawson, R. J. P. and Locher, K. P. (2007) Structure of the multidrug ABC transporter Sav1866 from Staphylococcus aureus in complex with AMPPNP. FEBS Letts. 581, 935-938

6 Ward, A., Reyes, C. L., Yu, J., Roth, C. B. and Chang, G. (2007) Flexibility in the $A B C$ transporter MsbA: Alternating access with a twist. Proc. Natl. Acad. Sci. USA. 104, 19005-19010

7 Jin, M. S., Oldham, M. L., Zhang, Q. and Chen, J. (2012) Crystal structure of the multidrug transporter P-glycoprotein from Caenorhabditis elegans. Nature. 490, 566-569

8 Loo, T. W. and Clarke, D. M. (2013) Drug rescue distinguishes between different structural models of human P-glycoprotein. Biochemistry. 52, 7167-7169

9 Szewczyk, P., Tao, H., McGrath, A. P., Villaluz, M., Rees, S. D., Lee, S. C., Doshi, R., Urbatsch, I. L., Zhang, Q. and Chang, G. (2015) Snapshots of ligand entry, malleable binding and induced helical movement in Pglycoprotein. Acta Cryst. D. 71, 732-741

10 Ward, A. B., Szewczyk, P., Grimard, V., Lee, C.-W., Martinez, L., Doshi, R., Caya, A., Villaluz, M., Pardon, E., Cregger, C., Swartz, D. J., Falson, P. G., Urbatsch, I. L., Govaerts, C., Steyaert, J. and Chang, G. (2013) Structures of P-glycoprotein reveal its conformational flexibility and an epitope on the nucleotide-binding domain. Proc. Natl. Acad. Sci. USA. $110,13386-13391$

11 Li, J., Jaimes, K. F. and Aller, S. G. (2014) Refined structures of mouse Pglycoprotein. Protein Sci. 23, 34-46

12 Chufan, E. E., Kapoor, K., Sim, H.-M., Singh, S., Talele, T. T., Durell, S. R. and Ambudkar, S. V. (2013) Multiple transport-active binding sites are available for a single substrate on human P-Glycoprotein (ABCB1). PLoS ONE. 8, e82463

$13 \mathrm{Ma}, \mathrm{J}$. and Biggin, P. C. (2013) Substrate versus inhibitor dynamics of Pglycoprotein. Prot. Struc. Func. Gen. 81, 1653-1668

14 O'Mara, M. L. and Mark, A. E. (2012) The effect of environment on the structure of a membrane protein: P-glycoprotein under physiological conditions. J. Chem. Theory Comput. 8, 3964-3976

15 Hrycyna, C. A., Airan, L. E., Germann, U. A., Ambudkar, S. V., Pastan, I. and Gottesman, M. M. (1998) Structural flexibility of the linker region of 
human P-glycoprotein permits ATP hydrolysis and drug transport. Biochemistry. 37, 13660-13673

16 Sato, T., Kodan, A., Kimura, Y., Ueda, K., Nakatsu, T. and Kato, H. (2009) Functional role of the linker region in purified human P-glycoprotein. FEBS. 276, 3504-3516

17 Ferreira, R. J., Ferreira, M.-J. U. and dos Santos, D. J. V. A. (2013) Assessing the stabilization of p-glycoprotein's nucleotide-binding domains by the linker, using molecular dynamics. Mol. Inform. 32, 529-540

18 Moradi, M. and Tajkhorshid, E. (2013) Mechanistic picture for conformational transition of a membrane transporter at atomic resolution. Proc. Natl. Acad. Sci. USA. 110, 18916-18921

19 Aittoniemi, J., de Wet, H., Ashcroft, F. M. and Sansom, M. S. P. (2010) Asymmetric switching in a homodimeric $A B C$ transporter: a simulation study. PLoS Comp. Biol. 6, e1000762

20 Becker, J.-P., Van Bambeke, F. O., Tulkens, P. M. and Prévost, M. (2010) Dynamics and structural changes induced by ATP binding in SAV1866, a bacterial ABC exporter. J. Phys. Chem. B. 114, 15948-15957

21 Gyimesi, G., Ramachandran, S., Kota, P., Dokholyan, N. V., Sarkadi, B. and Hegedús, T. (2011) ATP hydrolysis at one of the two sites in ABC transporters initiates transport related conformational transitions. Biochim. Biophys. Act. Biomembranes. 1808, 2954-2964

22 Jones, P. M. and George, A. M. (2011) Molecular-Dynamics simulations of the ATP/apo state of a multidrug ATP-Binding Cassette transporter provide a structural and mechanistic basis for the asymmetric occluded state. Biophys. J. 100, 3025-3034

23 Oliveira, A. S., Baptista, A. M. and Soares, C. M. (2011) Conformational changes induced by ATP-hydrolysis in an ABC transporter: A molecular dynamics study of the Sav1866 exporter. Proteins: Struct. Func. Bioinf. 79, 1977-1990

24 St-Pierre, J.-F., Bunker, A., Róg, T., Karttunen, M. and Mousseau, N. (2012) Molecular dynamics simulations of the bacterial ABC transporter SAV1866 in the closed form. J. Phys. Chem. B. 116, 2934-2942

25 Pajeva, I. K., Globisch, C. and Wiese, M. (2009) Comparison of the inward- and outward-open homology models and ligand binding of human P-glycoprotein. FEBS J. 276, 7016-7026

26 Pajeva, I. K., Globisch, C. and Wiese, M. (2009) Combined pharmacophore modeling, docking, and 3D QSAR studies of ABCB1 and ABCC1 transporter inhibitors. ChemMedChem. 4, 1883-1896

27 Palmeira, A., Vasconcelos, M. H., Paiva, A., Fernandes, M. X., Pinto, M. and Sousa, E. (2012) Dual inhibitors of P-glycoprotein and tumor cell growth: (Re)discovering thioxanthones. Biochem. Pharm. 83, 57-68

28 Yamaguchi, H., Kidachi, Y., Kamiie, K., Noshita, T. and Umetsu, H. (2012) Homology modeling and structural analysis of human P-glycoprotein. Bioinformation. 8, 1066-1074

29 Tajima, Y., Nakagawa, H., Tamura, A., Kadioglu, O., Satake, K., Mitani, Y., Murase, H., Regasini, L. O., da Silva Bolzani, V., Ishikawa, T., Fricker, G. and Efferth, T. (2014) Nitensidine A, a guanidine alkaloid from Pterogyne nitens, is a novel substrate for human ABC transporter ABCB1. Phytomed. 21, 323-332 
30 Klepsch, F., Chiba, P. and Ecker, G. F. (2011) Exhaustive sampling of docking poses reveals binding hypotheses for propafenone type inhibitors of P-glycoprotein. PLoS Comput. Biol. 7, e1002036

31 Jabeen, I., Wetwitayaklung, P., Klepsch, F., Parveen, Z., Chiba, P. and Ecker, G. F. (2011) Probing the stereoselectivity of P-glycoproteinsynthesis, biological activity and ligand docking studies of a set of enantiopure benzopyrano[3,4-b][1,4]oxazines. Chem. Commun. 47, 25862588

32 Jabeen, I., Pleban, K., Rinner, U., Chiba, P. and Ecker, G. F. (2012) Structure-activity relationships, ligand efficiency, and lipophilic efficiency profiles of benzophenone-type inhibitors of the multidrug transporter PGlycoprotein. J. Med. Chem. 55, 3261-3273

33 Pajeva, I. K., Sterz, K., Christlieb, M., Steggemann, K., Marighetti, F. and Wiese, M. (2013) Interactions of the multidrug resistance modulators tariquidar and elacridar and their analogues with P-glycoprotein. ChemMedChem. 8, 1701-1713

34 Jara, G. E., Vera, D. M. A. and Pierini, A. B. (2013) Binding of modulators to mouse and human multidrug resistance P-glycoprotein. A computational study. J. Mol. Graph. Model. 46, 10-21

35 Singh, D., Godbole, M. and Misra, K. (2013) A plausible explanation for enhanced bioavailability of P-gp substrates in presence of piperine: simulation for next generation of P-gp inhibitors. Journal of Molecular Modeling. 19, 227-238

36 Xu, X., Li, R., Ma, M., Wang, X., Wang, Y. and Zou, H. (2012) Multidrug resistance protein $\mathrm{P}$-glycoprotein does not recognize nanoparticle C60: experiment and modeling. Soft Matter

37 Zhang, J., Sun, T., Liang, L., Wu, T. and Wang, Q. (2014) Drug promiscuity of P-glycoprotein and its mechanism of interaction with paclitaxel and doxorubicin. Soft Matter. 10, 438-445

38 Prajapati, R., Singh, U., Patil, A., Khomane, K., Bagul, P., Bansal, A. and Sangamwar, A. (2013) In silico model for P-glycoprotein substrate prediction: insights from molecular dynamics and in vitro studies. J. Comp. Aided Mol. Des. 27, 347-363

39 O'Mara, M. L. and Tieleman, D. P. (2007) P-glycoprotein models of the apo and ATP-bound states based on homology with Sav1866 and MalK. FEBS Letts. 581, 4217-4222

40 Wise, J. G. (2012) Catalytic transitions in the human MDR1 Pglycoprotein drug binding sites. Biochemistry. 51, 5125-5141

41 Brewer, F. K., Follit, C. A., Vogel, P. D. and Wise, J. G. (2014) In silico screening for inhibitors of P-glycoprotein that target the nucleotide binding domains. Mol. Pharm. 86, 716-726

42 Pajeva, I. K., Hanl, M. and Wiese, M. (2013) Protein contacts and ligand binding in the inward-facing model of human P-Glycoprotein. ChemMedChem. 8, 748-762

43 Zeino, M., Saeed, M. M., Kadioglu, O. and Efferth, T. (2014) The ability of molecular docking to unravel the controversy and challenges related to Pglycoprotein-a well-known, yet poorly understood drug transporter. Invest. New Drugs. 32, 618-625 
44 Pan, L. and Aller, S. G. (2015) Equilibrated atomic models of outwardfacing p-glycoprotein and effect of atp binding on structural dynamics. Sci. Rep. 5

45 Subramanian, N., Condic-Jurkic, K., Mark, A. E. and O'Mara, M. L. (2015) Identification of possible binding sites for morphine and nicardipine on the multidrug transporter P-glycoprotein using umbrella sampling techniques. Journal of Chemical Information and Modeling

46 Klappe, K., Hummel, I., Hoekstra, D. and Kok, J. W. (2009) Lipid dependence of $\mathrm{ABC}$ transporter localization and function. Chem. Phys. Lipids. 161, 57-64

47 Shen, M. Y. and Sali, A. (2006) Statistical potential for assessment and prediction of protein structures. Protein Sci. 15, 2507-2524

48 Benkert, P., Biasini, M. and Schwede, T. (2011) Toward the estimation of the absolute quality of individual protein structure models. Bioinformatics. 27, 343-350

49 Kodan, A., Yamaguchi, T., Nakatsu, T., Sakiyama, K., Hipolito, C. J., Fujioka, A., Hirokane, R., Ikeguchi, K., Watanabe, B., Hiratake, J., Kimura, Y., Suga, H., Ueda, K. and Kato, H. (2014) Structural basis for gating mechanisms of a eukaryotic P-glycoprotein homolog. Proc. Natl. Acad. Sci. USA. 111, 4049-4054

50 Wen, P.-C., Verhalen, B., Wilkens, S., McHaourab, H. S. and Tajkhorshid, E. (2013) On the origin of large flexibility of P-glycoprotein in the inwardfacing state. J. Biol. Chem. 288, 19211-19220

51 Aller, S. G., Yu, J., Ward, A., Weng, Y., Chittaboina, S., Zhuo, R., Harrell, P. M., Trinh, Y. T., Zhang, Q., Urbatsch, I. L. and Chang, G. (2009) Structure of P-glycoprotein reveals a molecular basis for poly-specific drug binding. Science. 323, 1718-1722

52 Hohl, M., Briand, C., Gr ${ }^{\circ}$ tter, M. G. and Seeger, M. A. (2012) Crystal structure of a heterodimeric $A B C$ transporter in its inward-facing conformation. Nat Struct Mol Biol. 19, 395-402

53 Shintre, C. A., Pike, A. C. W., Li, Q., Kim, J.-I., Barr, A. J., Goubin, S., Shrestha, L., Yang, J., Berridge, G., Ross, J., Stansfeld, P. J., Sansom, M. S. P., Edwards, A. M., Bountra, C., Marsden, B. D., von Delft, F., Bullock, A. N., Gileadi, O., Burgess-Brown, N. A. and Carpenter, E. P. (2013) Structures of ABCB10, a human ATP-binding cassette transporter in apo- and nucleotide-bound states. Proc. Natl. Acad. Sci. USA. 110, 9710-9715

54 Choudhury, H. G., Tong, Z., Mathavan, I., Li, Y., Iwata, S., Zirah, S., Rebuffat, S., van Veen, H. W. and Beis, K. (2014) Structure of an antibacterial peptide ATP-binding cassette transporter in a novel outward occluded state. Proc. Natl. Acad. Sci. USA. 111, 9145-9150 
Table 1. Overview of P-gp homologue crystal structures.

\begin{tabular}{|c|c|c|c|c|c|c|}
\hline Protein & PDB & $\begin{array}{l}\text { Resolution } \\
(\AA)\end{array}$ & $\begin{array}{c}\text { Distance between } \\
\text { NBDs }(\AA)\end{array}$ & $\begin{array}{c}\text { Sequence } \\
\text { identity }\end{array}$ & Conformation & Reference \\
\hline S.aureus Sav1866 & $2 \mathrm{HYD}$ & 3.0 & 8.8 & $34 \%$ & Outwards open & {$[5]$} \\
\hline V. cholera MsbA & $3 B 5 X$ & 5.5 & & & Inwards closed & [6] \\
\hline S. typhimurium MsbA & 3B60 & 3.7 & & & Outwards open & [6] \\
\hline T. maritima TM287/288 & 3QF4 & 2.9 & 15.6 & $30 \%$ & Inwards open & [52] \\
\hline C. elegans $\mathrm{ABCB} 1$ & $4 \mathrm{~F} 4 \mathrm{C}$ & 3.4 & 40.4 & $46 \%$ & Inwards open & {$[7]$} \\
\hline Mouse ABCB1 & $4 \mathrm{KSB}$ & 3.8 & 38.0 & $87 \%$ & Inwards open & {$[10]$} \\
\hline Human ABCB10 & $3 Z D Q$ & 2.85 & 24.3 & $37 \%$ & Inwards open & {$[53]$} \\
\hline E. coli McjD & 4PL0 & 2.7 & 8.4 & $34 \%$ & Outwards occluded & {$[54]$} \\
\hline
\end{tabular}

Crystal structures of P-gp homologues. The distance between NBDs is represented by the distance of the N $\zeta$ atom on the Lys of the Walker A motif in NBD1 and the Ca atom of the Ser in the Signature Motif in NBD2. 
Table 2. Overview of human homology models of P-gp

\begin{tabular}{lcc}
\hline Protein & PDB & QMEAN Z-sı \\
\hline Sav1866 & 2HYD & -2.34 \\
ABCB1 (mouse) & $3 G 5 \mathrm{U}$ & -2.61 \\
ABCB1 (C. elegans) & $4 \mathrm{~F} 4 \mathrm{C}$ & -1.9 \\
ABCB1 (mouse) & $4 \mathrm{KSB}$ & -2.46 \\
ABCB10 (Human) & $3 \mathrm{ZDQ}$ & -2.5 \\
CmABCB1 (C. merolae) & $3 \mathrm{WME}$ & -2.14 \\
ABCB1 (Mouse refined) & $4 \mathrm{M} 1 \mathrm{M}$ & -1.86 \\
\hline
\end{tabular}

A QMEAN Z-score of less than -5 is taken as an indication there could be sc problematic with the structure [48]. 


\section{Figures}

\section{Figure 1| The structure and conformational states of P-gp homologues}

(A) Overall architecture of P-gp and its homologues. The pseudo two-fold symmetry is highlighted by the different colours. (B) Structures of homologues have been solved in different states, which are thought to reflect different stages of the transport cycle. (C) The majority of structures have been solved in the inward-open conformation, but there is huge variation in the separation of the NBDs (see Table 1).

\section{Figure 2 | The dynamics of P-glycoprotein in a model membrane}

(A) Illustration of the simulation box showing the human homology model from the refined mouse crystal structure $(4 \mathrm{M} 1 \mathrm{M})$ in a POPC bilayer with counter ions and water and the protein coloured according to the topology diagram in the top panel. (B) RMSD of Ca atoms of human homology models from three different templates; 4F4C (C. elegans): purple line, 3WME (C. merolae): blue line and 4M1M (refined mouse): green line (C) The distance between NBDs is represented by the distance of the $\mathrm{N} \zeta$ atom on the Lys of the Walker A motif in NBD1 and the Ca atom of the Ser in the Signature Motif in NBD2. Colours as in (B).

\section{Figure 3 | Substrate entrance pathways}

(A) Cartoon depicting how compounds might access the central drug-binding site. The substrates of P-gp have been proposed to reach the central binding site either through the lipid bilayer (the so-called "hydrophobic vacuum cleaner" model) or via the cytoplasm. (B) The homology model suggests that access to the central binding site could occur through an intra-membrane entrance portal (orange box) or a cytoplasmic entrance portal (blue box). In MD simulations (100 ns), the former tends to close, while the latter remains available for substrate passage throughout. 
Figure 1. Domicevica and Biggin

A

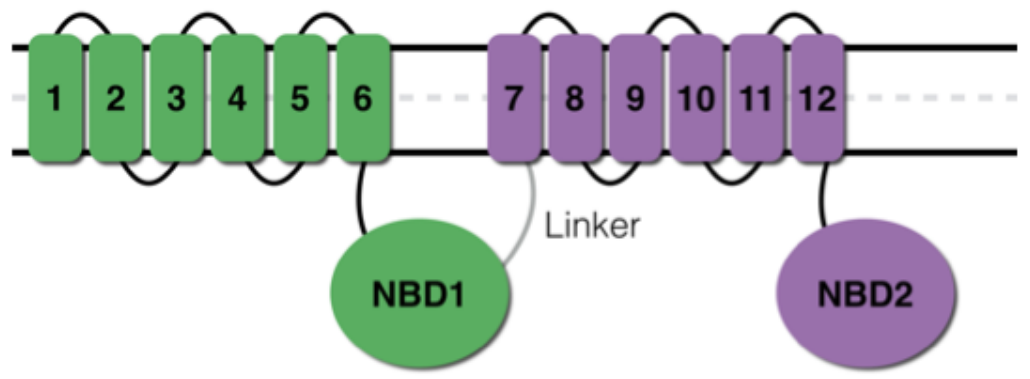

B

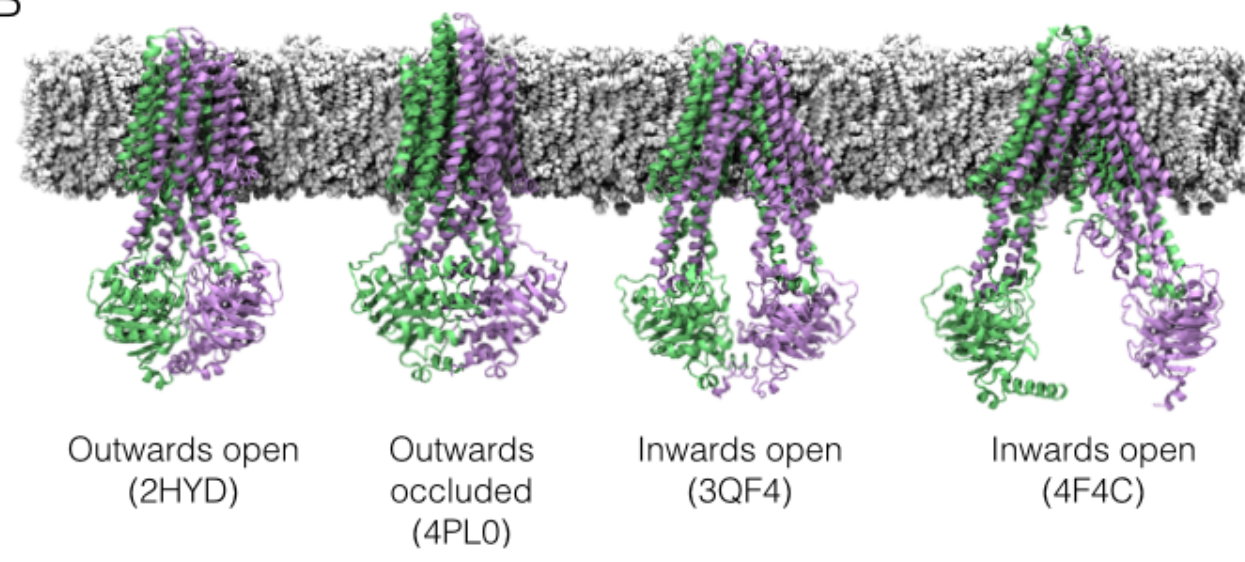

C

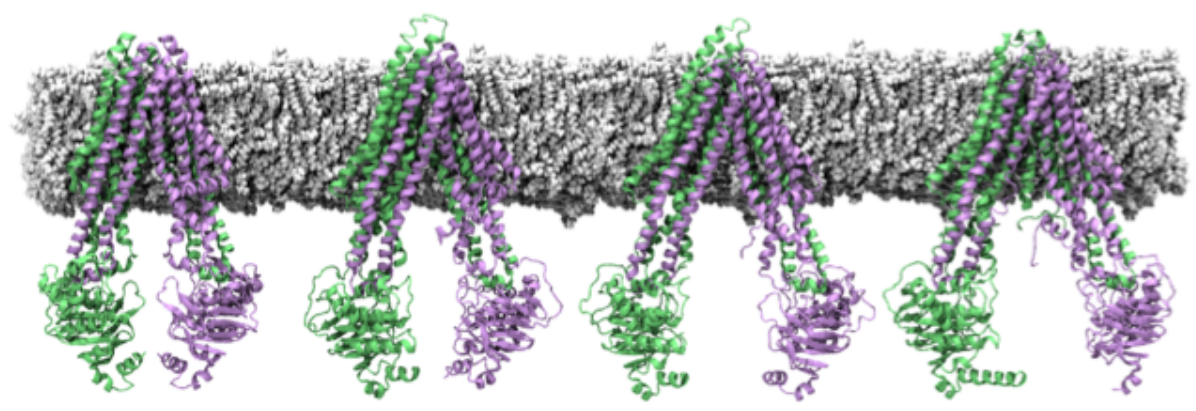
C. merolae
Mouse (refined)
Mouse
3WME
$4 \mathrm{M} 1 \mathrm{M}$
4KSB
C. elegans
$4 \mathrm{~F} 4 \mathrm{C}$ 
Figure 2. Domicevica and Biggin

A
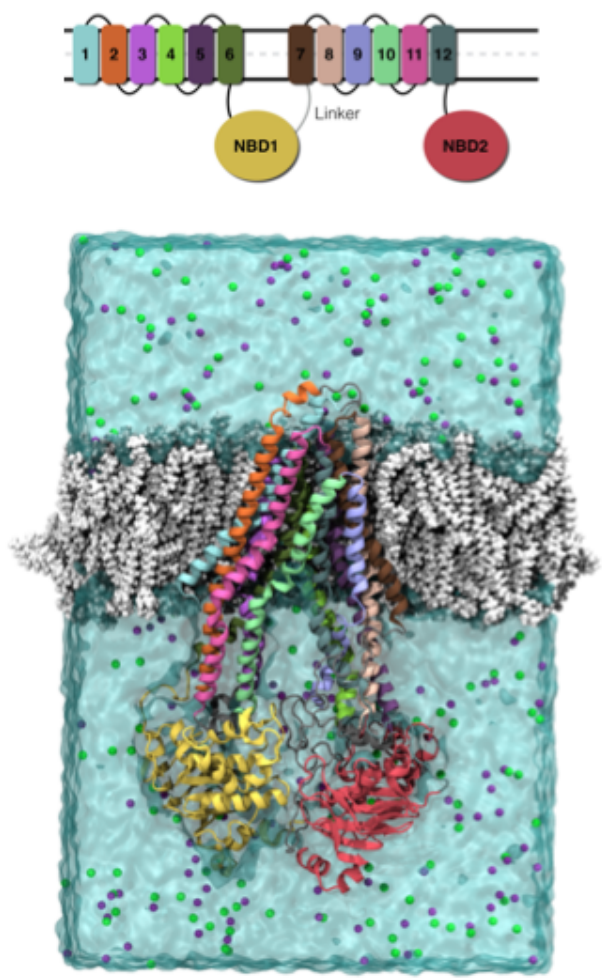

B RMSD

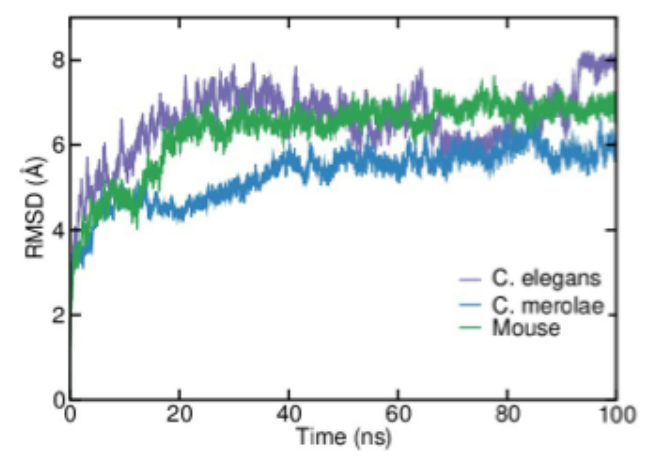

C

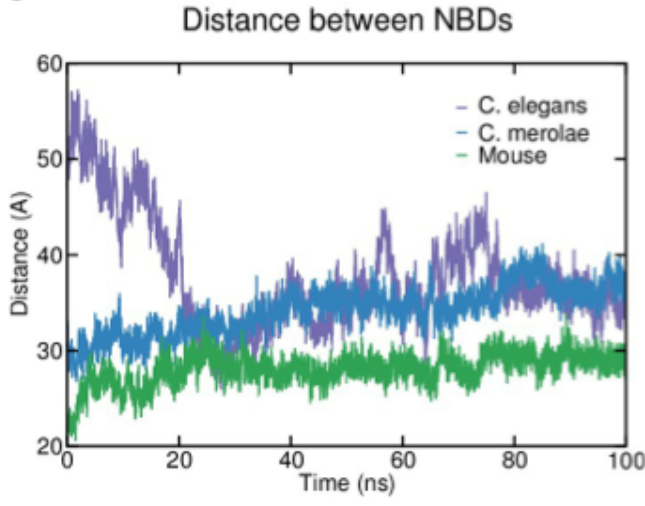


Figure 3. Domicevica and Biggin

A

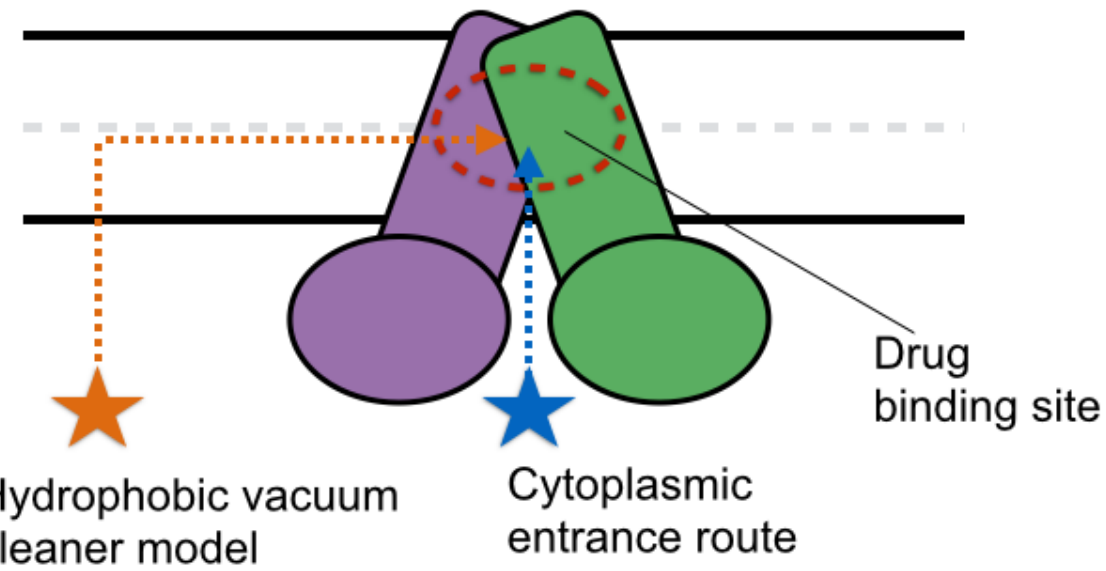

B

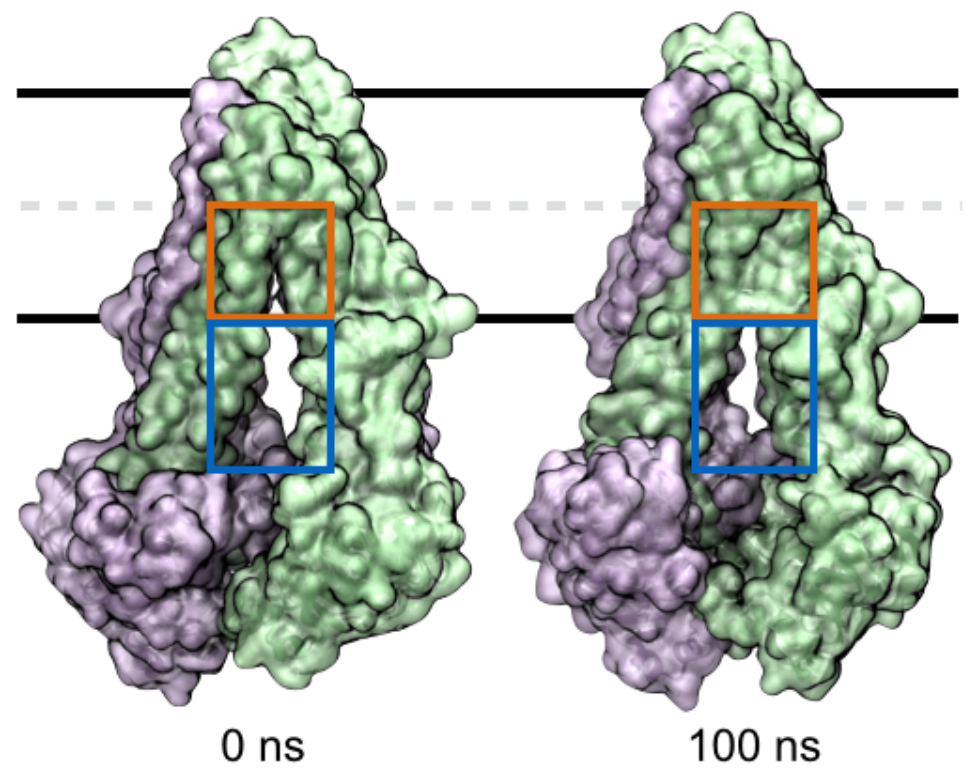

